\title{
A NEVER-ENDING STORY OF RHEUMATOID ARTHRITIS
}

\author{
BALJIT K. ${ }^{*}$, QADRIE Z. L. ${ }^{2}$, AMIT B. ${ }^{2}$, GAUTAM S. P. ${ }^{3}$ \\ ${ }^{* 1,2,3}$ Department of Pharmacology, CT Institute of Pharmaceutical Sciences, Jalandhar \\ Email: bk0173493@gmail.com
}

Received: 30 Oct 2019, Revised and Accepted: 18 May 2020

\begin{abstract}
There are distinct Rheumatic disorders, still Rheumatoid arthritis (RA) is believed to be very prevailing. RA is an empathic disorder described over integral redness, constant inflammation, and the existence of auto-antibodies. In RA, inflammation in joints, loss of motion of joint stiffness, joint tenderness are most common in patients. Deformity of joints can be prevented by early diagnosis and treatment. The extremity of the disease can be reduced by combining the drugs and improved weight more profiled than single medication. Treat-to-target progress results in a superior-conclusion in RA, and the ACR, EULAR, and other specialized systems have supported treat-to-target as a basic curative strategy for RA. The novel methods used in RA have upgraded the development of the disorder and maximum people helpful in cancellation of clinical manifestations if the identification of disorder takes place before time. This review article is written after studying most of the journal's articles, which were published between $1997-2019$.
\end{abstract}

Keywords: Rheumatoid arthritis, Pathology, Autoimmune inflammation, Auto-antibodies

(C) 2020 The Authors. Published by Innovare Academic Sciences Pvt Ltd. This is an open access article under the CC BY license (http://creativecommons.org/licenses/by/4.0/) DOI: http://dx.doi.org/10.22159/ijpps.2020v12i7.37438. Journal homepage: https://innovareacademics.in/journals/index.php/ijpps

\section{INTRODUCTION}

The RA is an erythrogenic disorder that causes persistent inflamed synovial membrane and thus collapses in the joints [1]. The synovial small and large joints such as fingers, elbows, shoulders, knees, and ankles are mainly included. The hardening and hyperplasia of a synovial line, along with permanent inflammation, are common symptoms of RA, causing severe disability and early death [2]. This autoimmune disease causes continuous joint destruction and decreased working ability and increase the death rate [3-5]. RA affects about $1 \%$ of the adult population worldwide, which is a big public health problem and social concern $[6,7]$. The epidemiological experiments showed that the popularity of RA is $0.5 \%-1.0 \%$. Hence, an estimated 11 million people could be affected by RA in India. About $75 \%$ of cases of RA related to autoantibodies, like rheumatoid factor, states that RA is an autoimmune disease [8]. Although the pathogenesis of RA remains not well known, the interaction between genetic and environmental factors are widely recognized for the initiation of RA [9-12]. RA causes intrinsic swelling, persevering tenosynovitis, and is regarded as the existence of autoantibodies; Citrullinated peptide and rheumatoid factor, which is also known to be an idiopathic disease. The disease is mostly occurring in females and the old persons and frequently strikes several joints leading to corrosion of articular surfaces [13]. RA is also linked with congenital difficulty, which includes acute myocardial infarction, pulmonary, mental, skeletal disorganization, and premature death. There is no remedy for RA; the advancement scheme is to facilitate a solution and get a low disease activity state (LDAS) [14].

\section{Ancient evidence of rheumatoid arthritis}

Despite the disorder, anciently, its designation RA is well known. During 1859, Sir Alfred Baring Garrod replacing the title to the medicinal published work of rheumatic gout. Miscellaneous interpretations of RA had disclosed formally by Heberden, Haygarth, Charcot and Brodie, and others $[15,16]$. This is in the same way medicinal thinking has become different over time, yet it also distinguishes a diseased state. The surveillance and the significance of manifestation required to clarify the situation of RA varied. The earliest sign of a disease related to recent RA put down Scribonius Largus, who explains a polyarthritis in women of 30 to $40 \mathrm{y}$ old [17]. The Roman emperor Constantine IX (980-1055 AD) was anciently the first famous person who experiences RA. The illustration of his illness develops in the historical account by Michael Psellus, who highlights chronic spondylitis entails the limb joints accomplished by acute muscle contraction, irregularities of hands and later incapability [18]. The genetic deposition is the beginning of RA. Genetical is a significant cause for the generation of RA because it more often occurs in families. Various environmental factors such as air pollution, history of smoking, coffee consumption have also stayed in the development of RA. The concept of the continual renewing of the natural immunity across climate moderately builds up autoimmune disease, which results in the advancement of RA. Asymptomatic inflammation is the later stage of RA, which leads to a generation of autoimmune. The rheumatic factor and inflammable markers are initially noticeable, proceeding to signs and detection of RA. Rheumatoid factor (RF) shows that existing earlier to the starting of RA [19]. The first symptoms of inflammation can be seen in its third phase of arthritis. During this phase, pain and inflammation of the joint occur, which is because of inflammatory cells such as lymphocytes and the synovial membrane are invaded by macrophages, which may lead to inflammation, movement and functional disability. Undifferentiated arthritis (UA) is an Early stage of inflammatory arthritis that occurs at different joints and it can diagnose within $6 \mathrm{w}$ to $1 \mathrm{y}$. About $30 \%$ of UA patients may lead to progress in RA. Irreversible joint damage, inflammation, and disability may be inhibited at this stage because UA is the turning point, which may lead to the development of RA. Chronic inflammation may lead to the severity of RA with irreversible destruction of joints through cartilage and bone erosion.

The stage at which RA can first be diagnosed is known as the fourth phase! The symptoms may seem within $6 \mathrm{w}$. The late course of RA is known as $5^{\text {th }}$ phase of RA and symptoms of this is characterized by the worse situation, the chronic inflammation of RA occurs during this phase causes more intense affliction to the affected joints and shoulder and hip joints. The later immune response has been influenced by environmental factors and genetic components and research has shown that different cells like T-cells, B-cells, synovitis and macrophages, which are taken a key regulator of immunological events throughout the year in RA [20]. The RA may be developed because of the interaction of environmental and genetic factors. Using oral contraceptive, breast-feeding, coffee/alcohol intake and birth weight irregularities are important environmental factors that are involved in the risk of progression of RA [21].

\section{Pathophysiological progressions from the old to the new world}

The traditional pathophysiology strategy is reliable, while it was established duration of therapy was expanded. It has many parts for 
a no. of years, as the certified therapy, while act upon investigated factors. The activation (stimulated by a hypothetical antigen) brings about lymphocyte growth and distinction, and as a result, the building of inflammatory cytokines such as IL-6, TNF- $\alpha$, IL-1, and IL17. These cytokines are corresponding to the activation of innate immunity constituents [22]. The HLA molecules stimulate the primary antigens to a T-cell antigen of $\mathrm{CD} 4$ cells by Co-stimulation of molecules like CD28-CD80/CD86 interaction. They serve as an anchor. The stimulation is not possible or not enough to trigger off downstream events without that cytokines. The mature T-cells also stimulate B-cells directly or indirectly. Finally, inflammatory cytokines especially IL- 6 activates the JAK/STAT pathway inhibitor which shows its effectiveness [23].

\section{Demonstrations related with rheumatoid arthritis}

The synovium is the first structure of a joint that is affected in the case of RA. The serious demonstrations of RA are listed into three that comprise cartilage, air passage, and circulatory system. Under the condition of cartilage demonstration, an entire system of bones affected in situ and intrinsic. At the local level, circumstances that raise osteoclasts resulted in a proliferation of bone structure, and these osteoclasts were liberated from inflammatory and fibroblastic pannus cells [24]. Primarily, RA patients were susceptible to bisphosphonate treatment for osteoporosis or avoiding glucocorticoid-mediated bone loss. Air passage demonstration of RA comprises cricoarytenoid arthritis, pulmonary fibrosis, and respiratory disorders, any unusual blockage in respiratory capability. Lung disorder is well known in RA cases primarily in male smokers. The probability of death occurs in about $40 \%$ of RA patients with congestive heart disease with a high extent of erythrogenic markers [25]. There is more risk of stroke and myocardial infraction about 2-fold increase in the patients of RA which are suffering from about $10 \mathrm{y}$ or more. It has been concluded that the patients of RA show more risk of having heart disease which can lead to increase mortality rates [26].

\section{Diagnosis of rheumatoid arthritis}

ACPA (Anti-Citrullinated Protein Antibodies) is the chief endogenous entities that originate throughout the early stage of the disease in RA patients. Although, the signs and symptoms of a patient at this phase lead to a dilemma in diagnosis. A doctor should start with a physical examination of the joints, mainly the small ones which include those of wrist, hand, feet, etc. to expose any swelling, redness, pain, stiffness, tenderness, body heat, and then examine muscle strength and reflexes. Notable inflammation, exactly synovitis, with not at all serious identification in different circumstances is an inherent mark of RA. After all, RA is designated by the synthesis of ACPA antibodies as opposed to the citrullinated proteins, these antibodies are dignified for its diagnosis [27]. This examination expresses the autoimmune response that gives rise to the sign of pain, swelling of joints, joint stiffness, etc. ACPA levels are expecting the inflammatory response in the future [28]. Although, only $60-70 \%$ of the population reveals high ACPA levels of RA ( $>20$ units $/ \mathrm{ml}$ ). The unavailability of ACPA in individuals does not show an absence of RA. Together with ACPA, a C-reactive protein test is also accomplished to establish the diagnosis. However, C-reactive protein levels are inaccurate and specific to rheumatoid arthritis. The complete Blood Count of an individual is also suggested to find anemic conditions related to RA. An alternative factor such as Rheumatoid factor (RF), is developed in $80 \%$ of the individuals with RA, in higher levels of 20 units $/ \mathrm{ml}$. The existence of RA is connected to expected rheumatoid nodules. The Creactive protein test and Erythrocyte Sedimentation Rate(ESR) test is supplemented to the ACPA and RF tests in diagnosing RA. To further access the situation of bone erosion in patients and work on the slow progression of the disability, diagnostic procedures such as X-rays, MRI, and Ultrasound are used [29]. A joint assay of An American College of Rheumatology and European League Against Rheumatism has granted Rheumatoid arthritis classification criteria 2010 , that targeted on premature identification of RA and approves for safeguard or termination of inflammatory reaction to break out the future drawbacks occurs due to RA [30]. Current approaches in the diagnosis have focused on the character of miRNA as an inherent indicator in RA [31]. These are the noncoding substances in plasma and synovial fluid, complete and safeguard from the operation of
RNAse available primarily. They govern distinct cell biology and mRNA steps. Similar plasma and synovial miRNAs reveal specialized extrusions and this can look out for analysis of RA and osteoarthritis. Plasma miR-132 helps in individualizing healthy persons from those with RA or osteoarthritis. Discrimination is achieved from synovial fluid miRNAs including miR-16, miR-146a, miR-155, and miR-223 which is expressed in considerable quantities in RA compared osteoarthritis. The interaction among these miRNAs and other scientific variables has been set up thus building miRNAs a valid source for RA diagnosis. To identify a separate biomarker for analysis of RA is difficult because of the fundamental complication in epidemiology with multiple entities collectively because of the ultimate RA demonstrations. Along with the abovementioned tests, $14-3-3 \eta$ protein complements the detection of RA with $77 \%$ susceptibility and 93\% precise [32]. Existence of IL-6, IL-13, IL-16, IL-22, and IL-33 cytokines represent synovial inflammation. Chemokine ligand 13(CXCL 13) is a biomolecule familiar to Blymphocytes while ficolin 3 is a compound from the innate humoral immune system [33-34]. In an attempt to find quantifiable, biomarkers in the early stages of RA, CD44vRA, and human galectin8 have arisen as a possibility [35]. The moderate surface glycoprotein CD44 is CD44vRA that conveys exactly the synovial cells in RA. CD44vRA is responsible for stimulating the creation of inflammatory molecules via the TLR-4 signaling pathway despite bringing out an immunogenic reaction. However, changed human galectin-8 is a ligand of high affinity for CD44vRA which is excreted by the synovial cells in RA. Interestingly galectin-8 is familiar to cause peripheral blood leucocytes and synovitis apoptosis. Another protein, acute-phase serum amyloid (A-SAA) is investigated to be the substitute marker of regional and innate inflammation. While its position in RA inflammation is not even verified, YKL-40 is a molecule of interest that may exhibit outstanding biomarkers [36]. It is also in control of 12 biomarkers used to determine the multi-biomarker disease activity (MBDA) score for evaluating the disease [37-38]. Other biomarkers for the delay stages of RA, its development and sign involve angiogenesis markers VEGF and angiopoietin-1, cartilage destruction indicators C-terminal, telopeptide of collagen I and II and MMP-3, etc. An open cohort study to encourage individualize treatment for Rheumatoid arthritis, axial spondylarthritis, psoriatic arthritis, and other inflammatory rheumatic diseases for a finding of current incorporation of biomarkers is in process with an established protocol [39]. Biomarkers predictive of the response to treatment are anti-MCV, cartilage oligomeric, matrix protein (COMP), Cal-protein, etc [40]. An exact union of biomarkers specified and delicate to RA is essential to identification followed by the management of RA.

\section{Therapy of rheumatoid arthritis}

The main aim of the treatment strategy in the management of RA is to reduce and controlling the activity of the disease. The latest medication for RA can be grouped into three postulates: diseasemodifying anti-rheumatic drugs (DMARDs), non-steroidal antiinflammatory drugs (NSAIDs), and glucocorticoids (GCs). In over 10 $y$, there has been a considerable approach to treat RA, together with an antagonistic work of disease-modifying antirheumatic drugs and the progress of immune therapies aim at relation to cells specific to RA immunopathogenesis. Verification manifests that DMARDs regulate inflammatory and immune reactions [41]. The superior planned use of factors that slow down disability development, known to be disease-modifying antirheumatic drugs or DMARDs, has to enhance end product in over $2 \mathrm{y}$. Laterally, initiation of DMARDs that kill proinflammatory molecules has spread the range of DMARD options available. DMARDs can bearranged into conventional synthetic DMARDs (csDMARDs) and specific targeted DMARDs. csDMARDs comprises methotrexate (MTX), sulfasalazine (SSZ), leflunomide (LEF), hydroxychloroquine (HCQ), cyclosporine, gold salts, d-penicillamine, and azathioprine (AZA). MTX is the keystone of RA treatment procedures and is mostly used. Selected remedy incorporates biologic DMARDs (bDMARDs) and targeted synthetic DMARDs (tsDMARDs) [42]. Biological agents such as etanercept (ETA), infliximab (INF), and tocilizumab (TCZ) can suppress tumor necrosis factor- $\alpha$ (TNF- $\alpha$ ) or stop interleukin- 6 receptor, have assigned vast improvements and transform the distant treatment of RA. However, inhibitors of the enzyme cyclooxygenase, which 
involves pain and inflammation [43]. Emerging from the new therapy for the management of RA is to improve the disease, activity, and quality of life of the patient. To reduce and preventing bone destruction, the biological DMARDs are used for treatment strategy. Before initiating the use of biological DMARDs, the proper evaluation of risk-benefit should be done and to prevent the risks of adverse effects like any malignancy or infection because of the use of biological DMARDs for treating RA [44]. Tumor necrosis factor inhibitors (TNFi) include adalimumab (ADA), certolizumab (CZP), etanercept (ETN), golimumab (GOL) and infliximab (IFX). Non-TNFi biologic agents include abatacept (ABA; a cytotoxic T lymphocyte antigen 4 [T-cell co-stimulation inhibitor]), anakinra (ANK; an antiinterleukin-1 receptor blocker), rituximab (RTX; an anti-CD20), and tocilizumab (TCZ; an anti-interleukin-6 receptor [IL-6R] blocker) [45]. Tocilizumab (TCZ) is a humanized anti-interleukin-6 (IL-6) receptor monoclonal antibody that is widely used to treat rheumatic arthritis (RA). When initially introduced, TCZ was administered by intravenous infusion. TCZ is approved worldwide and guidelines for RA treatment recommend TCZ as a first-line biological diseasemodifying anti-rheumatic drug (DMARDs). In recent years, the subcutaneous formulation of TCZ (TCZ-SC) has been developed in consideration of patient preference [46]. There are two explanations of IL-6R independent IL-6 signal transduction. The Epstein-Barr virus-induced gene 3 (EBI3), which is a subunit of the composite cytokines IL-27, IL35, and IL-39, is reported to mediate IL-6 transsignaling to gp130. The IL-6 inhibitor, but not the IL-6R inhibitor, can interfere with EBI3-dependent IL-6 signaling. Also, IL-30, the p28 subunit of the heterodimeric cytokine-27, binds IL6R and modulates inflammatory responses. It can impede the destructive process of RA and is often more efficient in controlling pain and disability than NSAIDs [47]. Thus, NSAIDs can relive partial pain but have no positive effect on slowing the progression of RA. NSAIDs can be beneficial in the early weeks after the onset of RA clinical symptoms and as a bridge treatment before the beginning of slowacting DMARDs. After oral administration, NSAIDs exhibit short halflife and thus it demands frequent and high dosing which easily causing causes gastrointestinal problems, platelet inhibition, and other adverse effects [48]. GCs are potent in suppressing inflammatory response and influencing immune cell's behavior; but their dose-dependent adverse effects are also distinct, especially only high doses of GCs can reach efficacy in RA [49]. Until now, although the therapeutic strategy of RA has changed from the use of traditional non-steroidal anti-inflammatory drugs (NSAIDs), glucocorticoids, and disease-modifying anti-rheumatic drugs (DMARDs) to bio-targeting agents of target cytokines, including tumor necrosis factor- $\alpha$ (TNF- $\alpha$ ), cyclooxygenase (COX), and interleukin (IL) and so on [50-52], these drugs will quiet have the burden of tremendous charges and several unwanted and severe adverse effects like humoral disturbances, GIT disturbances immunodeficiency. The global pharmaceutical industries targeted treating RA with adequate, inexpensive and less adverse effects which can be preferred by the RA patients and physicians. For a long duration, RA and fracture have been treated by using natural plant origin by china and different countries that have been significant for clinical progression and studied related to RA [53]. A formulation is developed for treating RA with methotrexate along with a combination of another drug. Glucocorticoid (GC) are more potent drugs for efficacious action and managing RA. GC can help treat RA as monotherapy and in combination with other drugs. The use of glucocorticoids for a long period may result in consideration of antiinflammatory activity and active against joint destruction. The glucocorticoids are effective for treating RA were prednisone, methyl-prednisone, hydrocortisone, triamcinolone, etc [54]. Janus kinase therapy is another approach for treating RA. Tofacitinib was Ist targeted DMARD recommended for treating RA along with baricitib which is an oral JAK inhibitor and it is later used. JAK inhibitors, TNF- $\alpha$ inhibitors, immunosuppressants, B-cell depletes, steroids, T-cell activation inhibitors are powerful for treating RA. The combinational treatment of RA was accomplished throughout the ancient ten years, although $1 / 3$ of patients of India prefer this combinational treatment for RA. The current process for treating RA is now changed from single-dose treatment to combinational treatment. The different dosage forms like tablets, parenteral, oral liquids, capsules, topical products, transdermal patches, pediatric/geriatric products are useable for the treating of RA. There are different topical dosage forms like paste, ointment, gel, cream, transdermal patches applicable for treating RA distribute medicament into the skin with the non-invasive method. The considerable inconvenience related to the typical dosage forms for the treating of RA were low patient conformity, a short half-life, less believability and low dissolution that may be enhanced by altering novel dosage forms [55]. Different tablets include Aspirin, Celecoxib, sulfasalazine, Leflunomide, Indomethacin conventional dosage forms in treating rheumatoid arthritis [56-57]. Capsules like Minocycline, Oxyproline, Cyclosporine, Tofacitinib, etc. Oral liquids include Azathioprine, Doxorubicin, etc. Topical (ointment gels) includes Hydroxychloroquine, Piroxicam, MTX, Ketoprofen, and Transdermal patches such as diclofenac sodium, Teriflunomide is also used as conventional antirheumatic drugs.

\section{Nanoparticles (NPS)}

NPS is tonic/opacifying agents, the most frequently described metallic NPs, liposomes, polymeric NPs and micelles manage effective transportation toward the therapy of RA. NPS has been administered through blood circulation via distinct procedures like adsorbing, receptor agonist extension, covalently coupled, and incorporation [58]. Drug delivery system of Non-Steroidal Anti-Inflammatory Drugs based delivery systems were mostly delivered for RA, that reduces pain suffering related to an initial phase of RA by its non-inflammation process in absence of coherent activity, also; which obstruct Cycloxygenase- 1 and Cycloxygenase- 2 enzymes that take part in a crucial characterization in the production of PGs. Drug carrying NPs systems were administered to treat inflamed synovial membrane [59]. Two NPs based on anti-rheumatic drugs involved curcumin, MTX, Tacrolimus, Dexamethasone and MTX, Celecoxib. The importance of NPs based formulation for the treating of RA manages enhancement of bioavailability, proliferate collection of medication at the diseased inflammation area, and lengthen the drug activity in the affected area. At the high level, these NPs based formulation also generates focusing possibility along with particular receptors site [60].

\section{NPS and nanocarriers}

\section{Interleukin-6 inhibition}

In 2017, Sarilumab approved by U. S. FDA is the newest biologic for treating RA [61]. IL-6 has a unique structure and a higher The NPs and nanocarriers are focusing on treating RA by a transformation of biological agents that permits targeted drug delivery. Through utilizing the amphiphilic essence of dextran sulphate, Heo et al. manufacture spherical NPs filled with methotrexate. This dextran sulphate NPs were collectively absorbed through activating macrophages with scavenger receptor class A mediated endocytosis. When the drug was administered systemically into CIA mice, the dextran sulphate NPs adequately gain in inflammation of joints entail their high selectivity at the affected area. However, the RA in CIA mice was improved by using methotrexate loaded dextran sulphate NPs which selectively improve the activity compared to free methotrexate taken lonely [62]. Nanotechnology is more effective and safer than biological DMARDs for treating RA. and it improves the pharmacokinetics of used biological DMARDS. affinity for receptors compared to tocilizumab. Sarilumab shows treating from mild to serious RA with incapability to react to methotrexate and can use with or without concomitant methotrexate. The recommended dose is $150-200 \mathrm{mg}$ SC for $2 \mathrm{w}$. The most common adverse effects reported include neutropenia, serious infection, hypersensitivity, and gastrointestinal perforation [63].

\section{Target synthetic dmards}

These are also called non-biological DMARDs. These are convenient (Tofacitinib) or in progressing target kinases involved in cell signalling. They are also called slow-acting antirheumatic drugs because of moderate action. JAKs are intracellular cytoplasmic tyrosine kinases. There are 4 JAKs available: JAK1, JAK2, JAK3, TyK2. The drugs are Tofacitinib, Baricitinib, Nilotinib [64].

\section{CD20 inhibition}

The monoclonal antibody is Rituximab which is a predominantly selected CD20 B cell. B cell inhibition is viewed to aid for RA by 
decreasing auto-antibody production and preventing antigen presentation to $\mathrm{T}$ cells. At $24 / 7 \mathrm{w}$, an exclusive way of rituximab associated with methotrexate treatment contributes remarkable and clinically relevant progress in disease activity in patients with active prevailing RA who had an appropriate response to one or more TNFi. Ocrelizumab is a humanized anti-CD20 antibody recommended by the US Foods and Drug Administration (FDA) for treating multiple sclerosis. Ocrelizumab influence considerable B cell depletion than rituximab. Ocrelizumab has illustrated the effectiveness of RA [65]. Ofatumumab exactly targets a membraneproximal epitope on the CD20 molecule different from other antiCD20 antibodies and rituximab and ocrelizumab and secure the epitope found on the large loop of CD20 which make a more durable B-cell depletion. Ofatumumab is related to higher binding of $\mathrm{C} 1 \mathrm{q}$ and more powerful adjunct-depending on cytotoxicity. Randomized clinical trials in RA reveals that ofatumumab to be efficient in methotrexate refractory patients and has clinically effective compared to rituximab at week 24 [66].

\section{Biosimilars}

Biosimilars are approved by the EMA or the FDA as having related efficiency and well-being as their particular biological inventor. Infliximab biosimilars (SB2 and CTP13) and the originator (Remicade) have related attainment of ACR20, depletion of radiographic progression, protection, and immunogenicity studies. Likewise, biosimilar Etanercept SB4 has similar effectiveness and protective description as the originator Enbrel [67]. A randomized, double-blind clinical trial shows that rituximab biosimilar PF05280586 has related effective and protective profiles as the originator rituximab. EULAR approves that biosimilars should be selected if they are appreciable of lower cost than the originator. The role of biosimilars in RA has been analysed away [68].

\section{Combinational therapy in rheumatoid arthritis: methotrexate and biologics}

It is revealed that patients who are using biologic and methotrexate at the same time may manifest satisfactory responses for curing in contrast to patients who use only biologic [69]. When TNF inhibitors are regulated in RA patients following an adverse reaction to methotrexate only, the alternative of combination therapy with methotrexate is chosen to TNF inhibitor only. Although, in patients with slight acute disease signs, therapy with a particular approach might be suitable. Besides, small scale precedence of tocilizumab in combination with methotrexate was to discover in contrast to tocilizumab only in patients without an acceptable response to methotrexate only [70]. In early RA patients who had not gained methotrexate, the clinical data of patients did not display a distinction between those who had taken Baricitinib monotherapy and those who were used with combinational Baricitinib and methotrexate treatment. However, patients administering combination therapy had only a small amelioration in radiographic consequence [71].

\section{Epigenetic treatment in rheumatoid arthritis}

Epigenetics is genetic changes of manifestation of genes, in which the nucleotide sequence of DNA remains unchangeable. DNA methylation, histone modifications, and microRNA related gene expression regulation are considered as the major implement of epigenetic procedures [72].

\section{Individualized pharmacogenetics in treating rheumatoid arthritis}

Pharmacogenomics is the examination and implementation of the particular genetic framework of patients to test the effect of drug therapy. Although the exceptional undifferentiated condition of the human genome, a small alternative in the genetic components regulate variability between individuals. The distinction in the singlenucleotide of the genome, while single nucleotide polymorphisms (SNPs), can alter the actions of coding protein. SNPs can occur in modified gene expression and thus influence the gene function [73].

\section{Rituximab}

RTX is a powerful therapy with an enduring expression on most of RA patients. However, the advantage of RTX to anti-TNF agents is uncertain, non-responders to TNF blockers have a probability to reach remission by RTX treatment. Although, low-dose RTX might be a substitute for a standard dose for patients who cannot permit or are at the possibility of inflammation growth/reactivation. The sign of response to RTX therapy is quite in its minor phase. Today, the diverse genetic factors, and the existence of specific cells or proteins, have been analysed as the indicator of RTX response. The inadequacy of research on the assurance of the safety of RTX risk before/during the pregnancy makes concerns about RTX safety at the time of conceiving. When moving to a majority of opinion, adhering to the latter delivery guidelines are approved [74].

\section{Treat to target in rheumatoid arthritis}

Treat-to-target is a modern progression in RA treatment that occupy exact checking of the illness and accommodating of executive action if a therapy is not enclosed. The ACR, European League against Rheumatism (EULAR), and the Asia Pacific League of Associations for Rheumatology (APLAR) have used the treat to target strategy in their instructions [75].

Treat-to-target has been well established as an assumption for the treating rheumatoid arthritis (RA) and encloses various well-defined constituents: selecting a target and a technique for calculating it; testing the target at a pre-indicative point; a dedication to change the treatment if the target is not attaining; and important measuring. A treat-to-target approach permits higher consequences to health care levels in RA, and the ACR, EULAR, and other professional companies have validated treat-to-target as a basic medicinal procedure for RA. However, details on the intensity to which treatto-target is engaged in the clinic are insufficient; it sounds that while several components of treat-to-target are mostly used, full performance persists unusually. Exceptional comprehensive interval to be directed incorporate how to choose the virtuous target for every patient, how rapidly to approach regardless the target has been accomplished, and then determine every successive treatment in documentation way [76].

\section{Alternative medicines}

Herbal medicines can be an alternative source of medicines for recovery from various diseases. For good relief, arthritic patients are increasingly seeking a natural (herbal) approach. Researchers are also showing interest in bioactive compounds derived from plants for RA treatment [77].

\section{Paederia scandens}

It is found in China and South Asian countries. It is used in the food and as medicine to treat arthritis and various gastrointestinal diseases. Iridoid glycosides, flavonoids, and volatile oil are the bioactive compounds. Among these, iridoid glycosides were found to inhibit the expression of TNF- $\alpha$, IL-1 $\beta$, and transforming growth factor-beta and exert a protective effect/8 against uric acid nephropathy and gouty arthritis in rats. Recently, a study by Xiao and colleagues explored the therapeutic effect of $P$. scandens using the type II collagen-induced arthritis (CIA) mouse model. The authors also focused on the modulation of the gut microbial community followed by $P$. scandens extract (PSE) treatment [78].

\section{Tripterygium wilfordii}

It is found in China. T. wilfordii extract was as effective as DMARD treatment in reducing the joints swellings and levels of CRP and erythrocyte sedimentation rate in RA patients [79].

\section{Probiotics}

Probiotics can improve the health of an individual when taken in the specified quantity. These probiotics are safe and also effective for RA. In a randomized double-blind, placebo-controlled trial on RA patients, treatment with a probiotic named Bifidobacterium supplementation resulted in reduced disease progression. Members of bifidobacterium and lactobacillus produce lactate and or acetate [80].

\section{P. histicola as a human gut-derived probiotic for rheumatoid arthritis}

Recently, a novel strain of P. histicola (MCI 001) was isolated from the human gut. Oral gavage of $P$. histicola in mice did not cause any 
intestinal pathology, even though it shifted the gut microbiome with an increased abundance of lactobacillus and sutterella. The studies with $P$. histicola suggest that it can be a probiotic for treating RA. Since $P$. histicola is endogenous to the human gut, it should have lower side effects. Another advantage of treating with known probiotics is that in case of side effects, patients can be treated with a targeted antibiotic [81].

\section{Aim for ra gene therapy: anti-and proinflammatory cytokines}

The advancement of inflammatory cytokines along fibroblast-like synovitis (FLSs) and activation of immune cells is showing a critical role in the growth and process of RA. The hindrance of proinflammatory cytokines and/or upregulation of antiinflammatory cytokines is the exceptional prevailing approach in RA therapy, which has been investigated across the beginning of the 2000s. The principal proinflammatory cytokines included in the pathogenesis of RA are tumour necrosis factor-alpha (TNF- $\alpha$ ), interleukin1 (IL-1), and IL-6. Minimizing the action of these cytokines beyond the management of $\mathrm{mABs}$ or soluble receptors lessens the expression of RA. Various mABs against TNF- $\alpha$ (infliximab, adalimumab, certolizumab pegol, etanercept, and golimumab), IL-1 (anakinra), and the IL-6 receptor (IL6-R), tocilizumab are accepted by the Food and Drug Administration (FDA). Anti-TNF treatment reveals good capability in combination with MTX, and this therapy is recommended as favourable RA therapy. The IL6-R inhibitor tocilizumab seems to be very efficient if taken alone [82]. Treatment with biologicals slows down the progress of RA and protect the functional report of involved joints. Although, a considerable amount of patients with RA (10-20\%) do not act in response to some current treatments [83].

\section{Herbal formulations as therapeutics for rheumatoid arthritis}

A few herbal extracts with favourable anti-inflammatory and antiarthritic response are explained in consecutive regions. The prevalence of medicinal plants is developing day by day because of the side effects of allopathic therapy. Herbal therapeutic plants have important sources of healing of human diseases since ancient times. Nowadays, $1 / 4$ th of the world population be able to trust traditional therapy, and $80 \%$ of the population depends on ancient herbal medicinal plants. Similarly, nowadays, maximum people living in different advanced countries depend on plant-derived therapy for firstly of primal health maintenance for minimizing or neglecting side effects [84].

\section{Triphala}

It is a used herbal formulation in Indian tradition which is found in fruits derived from three varieties: Embilica offficinalis (Indian gooseberry), Terminalia belerica (Chebulic myrobalan)[85]. Research has shown the anti-inflammatory and antioxidant effect of triphala in different arthritic models. In RA, Triphala shows to be anti-inflammatory action against adjuvant-induced arthritis and gouty arthritis models. It has shown to be an exceptional applicant for terminating cyclooxygenase-2 (COX-2) levels. These details show that triphala is an irreversible polyherbal formula with an effective curing effect next to several clinical symptoms in inflammatory diseases such as RA [86].

\section{Majoon ushba}

It is an Unani based polyherbal formulation obtained from 14 plant genus. It is widely used by Indian Unani formulation is to serve to manage an inflammatory state with least or negligible side effects [87].

\section{Trikatu}

It is a herbal extract composed of three commonly available crude drugs from the dried fruits of piper nigrum (Black pepper), Piper Longum (long pepper), and Zingiber officinalis (Ginger) in an equal ratio of $1: 1: 1(\mathrm{w} / \mathrm{w})$. Various details manifest the capability of Trikatu in managing inflammatory disorders such as RA. It slows down the making of inflammatory cytokines, pain mediators, osteoclastogenic factors in adjuvant-induced arthritis [88].

\section{Withania somnifera extract}

It's also known as Ashwagandha, which is a winter cherry native to the Solanaceae family that is used in managing a greater diversity of disease including asthma, diabetes, hypertension, stress, arthritis disease and cancer $[89,90]$.

\section{Barberry extract}

Barberry is an evergreen herb with various b. activities and tradition component used in plant-based therapy. This extract and its vital $b$. component $B B R$ has been remedially investigated in several inflammatory diseases and RA. $B B R$ is well known to influence the unusual cell activity in an inflammatory-microenvironment like RA. In particular, $B B R$ terminates myeloid-derived dendritic cells, which leads to mediate inflammation in RA, by stimulating the cell apoptosis. Therefore, the barberry extracted chief bioactive compound $B B R$ used as novel medicine for managing RA [91].

\section{Tripterygium wilfordii extract}

It is found in china and also known as 'thunder god wine'. It is commonly used for managing a greater variety of autoimmune disorders and inflammatory diseases. Various details show the consequence of this extract in the management inflammatory state of RA. It has approved to aid suffering while removing the damp and inflammation of the joint space [92].

\section{Angelica sinensis}

It is an equatorial herb. It belongs to Apiaceae family. It has latterly described in preventing RA. It also has been verified that A. Sinensis extract terminates osteoclast divergence of bone marrow monocytes/macrophages along with the deplete of Nfatc, c-Foc, CJun, TRAP, and OSCAR [93].

\section{Rosa multiflora}

It is a scrambling plant estimated around $3-5 \mathrm{~cm}$ in length along with leaves and feathered petals. The extract of plant $R$. multiflora has been entirely beneficial as a nutritional additive in managing broad diversity for an illness like acid, indigestion, flu, affliction, RA, swelling and osteoarthritis [94].

Various bioactive compounds available in herbal systems of plant basis manifest positive results in erythrogenic disease, specifically RA. They are obtained from native herb base, producing the system as a constituent of native nutrients absorption. Various researches show that formation has a greater replacement than the directly advised medicaments, which has a less adverse effect. Finally, this appears to reveal the herbal formulations and these bioactive compounds to be tested as a recommended substitute in managing RA [95].

\section{CONCLUSION}

Various compounds are available which are shown to have a favourable effect on inflammatory diseases such as RA. Various studies are shows the evidence for favourable alternatives to now approved drugs, with least or negligible side effects. This review shows the effect of different allopathic medications and herbal drugs to be tested as progressing alternatives for the treatment of rheumatoid arthritis. Hence, early diagnosis along with individualized treatment shall produce RA management in patients much easier.

\section{ACKNOWLEDGMENT}

Authors are thankful to the management and other teaching faculty of CT Institute of Pharmaceutical Sciences, Jalandhar for providing necessary library support and encouragement to compile the work.

\section{FUNDING}

Nil

\section{AUTHORS CONTRIBUTIONS}

All the authors have contributed equally.

\section{CONFLICT OF INTERESTS}

\section{Declared none}

\section{REFERENCES}

1. Pan T, Cheng T, Jia Y, Li P, Li F. Anti-rheumatoid arthritis effects of traditional Chinese herb couple in adjuvant-induced arthritis in rats. J Ethnopharmacol 2017;205:1-7. 
2. Smolen JS, Aletaha D, Barton A, Burmester GR, Emery P, Firestein A GS, et al. Rheumatoid arthritis. Nat Rev Dis Primers 2018;4:18001.

3. Abbasi M, Mousavi M. Strategies toward rheumatoid arthritis therapy; the old and the new. J Cell Physiol 2019;234:10018-31.

4. Wehr P, Purvis H, Law SC, Thomas R. Dendritic cells, T cells and their interaction in rheumatoid arthritis. Clin Exp Immunol 2019;196:12-27.

5. Van der Woude D, van der Helm-van Mil AHM. Update on the epidemiology, risk factors, and disease outcomes of rheumatoid arthritis. Best Pract Res Clin Rheumatol 2018;32:174-87.

6. Bourne AM, Johnston RV, Cyril S, Hill, C Hiller, SJ Kamper, et al. Scoping review of priority setting of research topics for musculoskeletal conditions. Br Med J Open 2018;8:e023962.

7. Okada Y, Eyre S, Suzuki A, Kochi Y, Yamamoto K. Genetics of rheumatoid arthritis: 2018 status. Ann Rheum Dis 2018;10:1-8.

8. Viatte S, Barton A. Enetics of rheumatoid arthritis susceptibility, severity, and treatment response. Semin Immunopathol 2017;39:395-408.

9. Frank Bertoncelj M, Klein K, Gay S. Interplay between genetic and epigenetic mechanisms in rheumatoid arthritis. Epigenomics 2017; 9:493-504.

10. Belbasis L, Dosis V, Evangelou E. Elucidating the environmental risk factors for rheumatic diseases: an umbrella review of meta-analyses. Int J Rheum Dis 2018;21:1514-24.

11. Jalil SF, Arshad M, Bhatti A, Ahmad J, Akbar F, Ali S. Rheumatoid arthritis: what have we learned about the causing factors? Pak J Pharm Sci 2016;29:629-45.

12. Bernstein, Elana J, Mandl LA. Changing incidence of orthopedic surgery in rheumatic disease: contributing factors. Curr Rheumatol Rep 2013;15:365-78.

13. Ometto F, Botsios C, Raffeiner B. Methods used to assess remission and low disease activity in rheumatoid arthritis. Autoimmun Rev 2010;9:161-4.

14. Forestier J. Heberden oration, 1962: three french pioneers in rheumatology. Ann Rheum Dis 1963;22:63-70.

15. Appelboom T, Ehrlich GE. Historical note: the concept of gout in 1880. Arthritis Rheum 1998:41:1511-2.

16. Copeman WSC, Berkeley MD, Angeles Los FRCP. A short history of gout and the rheumatoid arthritis. University of California Press 1964;7:145-236.

17. Aceves Avila FJ, Medina F, Fraga A. The antiquity of rheumatoid arthritis: a reappraisal. J Rheumatol 2001;28:751-7.

18. Rantapaa. Dahlqvist, Solbritt. Antibodies against cyclic citrullinated peptide and IgA rheumatoid factor predict the development of rheumatoid arthritis. Arthritis Rheum 2003;48:2741-9.

19. Yang M, Feng X, Ding J, Chang F, Chen X. Nanotherapeutics relieve rheumatoid arthritis. J Controlled Release 2017;252:108-24

20. McInnes IB, Schett G. Pathogenetic insights from the treatment of rheumatoid arthritis. Lancet 2017;389:2328-37.

21. Firestein GS, McInnes IB. Immunopathogenesis of rheumatoid arthritis. Immunity 2017;46:183-96.

22. Semerano L, Decker P, Clavel G, Boissier MC. Developments with investigational janus kinase inhibitors for rheumatoid arthritis. Expert Opin Investig Drugs 2016;25:1355-9.

23. Saag KG. Low-dose corticosteroid therapy in rheumatoid arthritis: balancing the evidence. Am J Med 1997;103:31S-9.

24. Movahedi M, Beauchamp ME. Risk of incident diabetes mellitus associated with the dosage and duration of oral glucocorticoid therapy in patients with rheumatoid arthritis. Arthritis Rheumatol 2016;68:1089-98.

25. Aggarwal R, Liao K, Nair R, Ringold S, Costenbader KH. Anticitrullinated peptide antibody assays and their role in the diagnosis of rheumatoid arthritis. Arthritis Rheum 2009;61:1472-83.

26. Pandya DT, Patel GC, Ambegaokar PP, Vyas ND. Comorbidity of cardiovascular diseases and rheumatoid arthritis. Asian J Pharm Clin Res 2017;10:72-7.

27. Kroot EJ, de Jong BA, van Leeuwen MA. The prognostic value of anti-cyclic citrullinated peptide antibody in patients with recent-onset rheumatoid arthritis. Arthritis Rheum 2000;43:1831-5.
28. Colebatch AN, Edwards CJ. EULAR recommendations for the use of imaging of the joints in the clinical management of rheumatoid arthritis. Ann Rheum Dis 2013;72:804-14.

29. Aletaha D, Neogi T. Rheumatoid arthritis classification criteria: an American College of Rheumatology/European League Against Rheumatism collaborative initiative. Arthritis Rheum 2010;62:2569-81.

30. Murata K, Yoshitomi H, Tanida S. Plasma and synovial fluid microRNAs as potential international biomarkers of rheumatoid arthritis and osteoarthritis. Arthritis Res Ther 2010;12:R86.

31. Gavrila BI, Ciofu C, Stoica V. Biomarkers in rheumatoid arthritis, what is new? J Med Life 2016;9:144-8.

32. Mc Ardle A, Flatley B, Pennington SR, FitzGerald O. Early biomarkers of joint damage in rheumatoid and psoriatic arthritis. Arthritis Res Ther 2015;17:141.

33. Srikantiah C. Current studies of biomarkers for the early diagnosis of rheumatoid arthritis. Curr Biomarker Findings 2014;4:107-19.

34. Golan I. Disease-specific biomarkers for early diagnosis of rheumatoid arthritis and other chronic arthritic conditions. MOJ Immunol 2016:3:83-5.

35. Ferreira JF, Ahmed Mohamed AA, Emery P. Glucocorticoids and rheumatoid arthritis. Rheum Dis Clin North Am 2016;42:33-7.

36. McDonough AK, Curtis JR, Saag KG. The epidemiology of glucocorticoid-associated adverse events. Curr Opin Rheumatol 2008;20:131-7.

37. Saag KG, Koehnke R. Low dose long-term corticosteroid therapy in rheumatoid arthritis: an analysis of serious adverse events. Am J Med 1994;96:115-23.

38. Nicolaides NC, Galata Z, Kino T, Chrousos GP, Charmandari E. The human glucocorticoid receptor: molecular basis of biologic function. Steroids 2010;75:1-12.

39. Vandevyver S, Dejager L, Libert C. On the trail of the glucocorticoid receptor into the nucleusand back. Traffic 2012;13:364-74.

40. McInnes IB, Schett G. Pathogenetic insights from the treatment of rheumatoid arthritis. Lancet 2017;389:2328-37.

41. Rein P, Mueller RB. Treatment with biologicals in rheumatoid arthritis: an overview. Rheumatol Ther 2017;4:247-61.

42. Badri W, Miladi K, Nazari QA, Greige Gerges H, Fessi H, Elaissari A. Encapsulation of NSAIDs for inflammation management: overview, progress, challenges and prospects. J Pharm 2016;515:757-73

43. Siebert S, Tsoukas A, RobertsonJ, McInnes I. Cytokines as therapeutic targets in rheumatoid arthritis and other inflammatory diseases. Pharmacol Rev 2015;67:280-309.

44. Razak Abdul Salmi, Bakry Makmor Mohd, Redzuan MD-Adyani. Management of rheumatoid arthritis: special consideration for biologic disease-modifying antirheumatic drugs. Asian J Pharm Clin Res 2018;11:47-53.

45. Ogata A, Kato Y, Higa S. IL-6 inhibitor for the treatment of rheumatoid arthritis: a comprehensive review. Mod Rheumatol 2019;29:258-67.

46. Petes C, Mariani MK, Yang Y. Interleukin (IL)-6 inhibits IL27and IL-30-mediated inflammatory responses in human monocytes. Front Immunol 2018;9:256.

47. Harirforoosh S, Asghar W, Jamali F. Adverse effects of nonsteroidal anti-inflammatory drugs: an update of gastrointestinal, cardiovascular and renal complications. J Pharm Pharm Sci 2014;16:821-47.

48. Yang M, Feng X, Ding J, Chang F, Chen X. Journal of controlled release: official. J Control Release Soc 2017;252:108.

49. Liu LL, Mao YY, Gao LB. Development of rheumatoid arthritis drugs in the world. Sci Technol Rev 2016;34:44-55.

50. Liu XT, Li Q. Advances in the treatment of rheumatoid arthritis. Adv Biomed 2015;15:1171-3.

51. Smolen JS, Aletaha D, Koeller M, Weisman MH, Emery P. New therapies for the treatment of rheumatoid arthritis. Lancet 2007;370:1861-74.

52. Lu S, Wang Q, Li G, Sun S, Guo Y, Kuang H. The treatment of rheumatoid arthritis using Chinese medicinal plants: from pharmacology to potential molecular mechanisms. J Ethnopharmacol 2015;176:177-206. 
53. Chandrappa M, Biswas S. Glucocorticoids in management of adult rheumatoid arthritis-current prescribing practices and perceptions of physicians in India: Glumar survey. Rheumatol Curr Res 2017;7:220.

54. Movahedi ME, Beauchamp. Risk of incident diabetes mellitus associated with the dosage and duration of oral glucocorticoid therapy in patients with rheumatoid arthritis. Arthritis Rheumatol 2016;68:1089-98.

55. Al-Rashed F, Calay D. Celecoxib exerts protective effects in the vascular endothelium via COX-2-independent activation of AMPK-CREB-Nrf2 signalling. Sci Rep 2018;8:6271.

56. Brown PM, Pratt AG, Isaacs JD. Mechanism of action of methotrexate in rheumatoid arthritis, and the search for biomarkers. Nat Rev 2016;12:731.

57. Comi G, Freedman L MS. Pooled safety and tolerability data from four placebocontrolled teriflunomide studies and extensions. Multiple Sclerosis Related Disorders 2016;5:97-104.

58. Behzadi S, Serpooshan V. Cellular uptake of nanoparticles: journey inside the cell. Chem Soc Rev 2017;46:4218-44.

59. Dolati S, Sadreddini S, Rostamzadeh D, Ahmadi M, Jadidi-Niaragh F, Yousefi M. Utilization of nanoparticle technology in rheumatoid arthritis treatment. Biomed Pharmacother 2016;80:30-41.

60. Kumar V, Leekha A, Tyagi A, Kaul A, Mishra AK, Verma AK. Preparation and evaluation of biopolymeric nanoparticles as drug delivery system in effective treatment of rheumatoid arthritis. Pharm Res 2017;34:654-67.

61. Heo R. Dextran sulfate nanoparticles as a theranostic nanomedicine for rheumatoid arthritis. Biomaterials 2017;131:15-26.

62. Aly AM, Furst DE. Update of sarilumab to treat rheumatoid arthritis based on randomized clinical trials: a systemic review. Expert Rev Clin Immunol 2017;13:741-52.

63. Fleischmann R, van Adelsberg J, Lin Y. Sarilumab and nonbiologic disease modifying antirheumatic drugs in patients with active rheumatoid arthritis and inadequate response to tumor necrosis factor inhibitors and inadequate response or intolerance to tumor necrosis factor inhibitors. Arthritis Rheumatol 2017:69:277-90.

64. Verden A, Dimbil M, Kyle R, Overstreet B, Hoffman KB. Analysis of spontaneous postmarket case reports submitted to the FDA regarding thromboembolic adverse events and JAK inhibitors. Drug Saf 2018;41:357-61.

65. Abushouk AI. Safety and efficacy of ocrelizumab in rheumatoid arthritis patients with an inadequate response to methotrexate or tumor necrosis factor inhibitors: a systematic review and meta-analysis. Rheumatol Int 2017;37:1053-64.

66. Pers YM, Jorgensen C. Perspectives of atumumab as CD20 targeted therapy in rheumatoid arthritis and other autoimmune diseases. Immunotherapy 2016;8:1091-6.

67. Emery P. A phase III randomised, double-blind, parallel-group study comparing SB4 with etanercept reference product in patients with active rheumatoid arthritis despite methotrexate therapy. Ann Rheum Dis 2017;76:51-7.

68. Kay J. Consensus-based recommendations for the use of biosimilars to treat rheumatological diseases. Ann Rheum Dis 2018;77:165-74.

69. Gabay C, Riek M, Scherer A, Finckh A. Effectiveness of biologic DMARDs in monotherapy versus in combination with synthetic DMARDs in rheumatoid arthritis: data from the swiss clinical quality management registry. Rheumatology 2015;54:1664-72.

70. Teitsma XM, Marijnissen AKA. Tocilizumab as monotherapy or combination therapy for treating active rheumatoid arthritis: a meta-analysis of efficacy and safety reported in randomized controlled trials. Arthritis Res Ther 2016;18:211.

71. Fleischmann R, Schiff M. Baricitinib, methotrexate, or combination in patients with rheumatoid arthritis and no or limited prior disease-modifying antirheumatic drug treatment. Arthritis Rheumatol 2017;69:506-17.

72. Karami J, Mahmoudi M. Promoter hypermethylation of BCL11B gene correlates with downregulation of gene transcription in ankylosing spondylitis patients. Genes Immunity 2017;18:170-5.

73. Gupta V, Sengupta M, Prakash J, Tripathy BC. Pharmacogenomics and pharmacogenetics. Basic Appl Aspects Biotechnol 2017;1:151-66.
74. Tavakolpour S, Alesaeidi S, Darvishi M. A comprehensive review of rituximab therapy in rheumatoid arthritis patients. Clin Rheumatol 2019;38:2977-94.

75. Ramiro S, Sepriano A, Chatzidionysiou K. Safety of synthetic and biological DMARDs: a systematic literature review informing the 2016 update of the EULAR recommendations for management of rheumatoid arthritis. Ann Rheum Dis 2016;76:1101-36.

76. Van Vollenhoven R. Treat-to-target in rheumatoid arthritis-are we there yet? Nat Rev Rheumatol 2019;15:180-6.

77. Choudhary M, Kumar V, Malhotra H. Medicinal plants with potential anti-arthritic activity. J Intercult Ethnopharmacol 2015;4:147-53.

78. Xiao M, Fu X, Ni Y. Protective effects of Paederia scandens extract on rheumatoid arthritis mouse model by modulating gut microbiota. J Ethnopharmacol 2018;226:97-104.

79. Wang J, Chen N, Fang L. A systematic review about the efficacy and safety of tripterygium wilfordii hook. f. preparations used for the management of rheumatoid arthritis. J Evid Based Complementary Altern Med 2018;2018:1-13.

80. Aoki R, Kamikado K, Suda W. A proliferative probiotic bifidobacterium strain in the gut ameliorates progression of metabolic disorders via microbiota modulation and acetate elevation. Sci Rep 2017;7:43522.

81. Balakrishnan B, Luckey D, Marietta E. Development of a realtime PCR method for quantification of Prevotella histicola from the gut. Anaerobe 2017;48:37-41.

82. Abbasi M, Mousavi MJ, Jamalzehi S, Alimohammadi R, Bezvan $\mathrm{MH}$, Mohammadi $\mathrm{H}$, et al. Strategies toward rheumatoid arthritis therapy: the old and the new. J Cell Physiol 2019;234:10018-31.

83. Olsen IC, Lie E, Vasilescu R, Wallenstein G, Strengholt S, Kvien TK. Assessments of the unmet need in the management of patients with rheumatoid arthritis: analyses from the NORDMARD registry. Rheumatology 2019;58:481-91.

84. Xu-zhao Li, Shuai nan-Zhung. Herbal compounds for rheumatoid arthritis: Literature review and informatics prediction. Phytother Res 2019;34:1-16.

85. Kalaiselvan S, Rasool M. Triphala herbal extract suppresses inflammatory responses in LPS-stimulated RAW 264.7 macrophages and adjuvant-induced arthritic rats via inhibition of NF-kappa B pathway. J Immune Toxicol 2016;13:509-25.

86. Peterson CT, Denniston K, Chopra D. Therapeutic uses of triphala in ayurvedic medicine. J Altem Complement Med 2017;23:23:607-14.

87. Lone AH, Ahmad T, Niyar AH. Clinical evaluation of efficacy of Majoon Ushba and roghane hindi in the management of psoriasis: a randomized single-blind, placebo-controlled study. J Ayurveda Int Med 2011;2:26-31.

88. Murunikkara V, Rasool MK. Trikatu, an herbal compound mitigates the biochemical and immunological complications in adjuvant induced arthritic rats. Int J Rheum Dis 2017;20:298308.

89. Shasmita, Rai MK, Naik SK. Exploring plant tissue culture in Withania somnifera (L.) Dunal: in vitro propagation and secondary metabolite production. Crit Rev Biotechnol 2018;38:836-50.

90. Dinesh P, Rsool M. Berberine, an isoquinolne alkalois suppresses TXNIP mediated NLRP3 inflammasome activation in MSU crystal stimulated RAW 264.7 macrophages through the upregulation of Nrf2 transcription factor and alleviates MSU crystal induced inflammation in rats. Int Immune Phamacol 2017;44:26-37.

91. Li H, Kong D, Xu Y. Tripterygium Wilfordii inhibits tonsillar IgA production by downregulating IgA class switching in IgA nephropathy. Oncotarget 2017;8:109027-42.

92. Ji P, Wei Y, Hua Y. A novel approach using metabolomics coupled with hematological and biochemical parameters to explain the enriching-blood effect and mechanism of unprocessed Angelica sinensis and its 4 kins of processed produts. J Ethnopharmacol 2018;211:101-16.

93. Nakamura N, Hirakawa H, Sato S, Otagaki S, Matsumoto S, Tabata S, et al. Genome structure of Rosa multiflora, a wild ancestor of cultivated roses. DNA Res 2018;25:113-21. 
94. Palani Dinesh, Mahaboobkhan Rasool. Herbal formulations and their bioactive components as dietary supplements for treating rheumatoid arthritis. Chapter 22; 2019. p. 385-99.
95. Nimesh S. Herbal drug is better than allopathic drug in the treatment of rheumatoid arthritis. Int J Pharmacogn 2018;5:539-45 